\title{
Two Stability Criteria for Benzenoid Hydrocarbons and Their Relation
}

\author{
Ivan Gutman,* Izudin Redžepović, Boris Furtula
}

\author{
Faculty of Science, University of Kragujevac, P. O. Box 60, 34000 Kragujevac, Serbia \\ * Corresponding author's e-mail address: gutman@kg.ac.rs
}

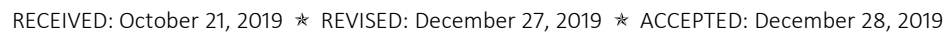

PROCEEDING OF THE $31^{\text {ST }}$ MATH/CHEM/COMP CONFERENCE, JUNE 10-14, 2019, DUBROVNIK, CROATIA

DEDICATED TO THE MEMORY OF DR. EDWARD CAMERON KIRBY

Abstract: A new, simple, relation is established between the total $\pi$-electron energy and the HOMO-LUMO gap, applicable to benzenoid hydrocarbons.

Keywords: total $\pi$-electron energy, highest occupied molecular orbital, HOMO-LUMO gap, McClelland inequality, Oboudi inequality.

\section{INTRODUCTION}

W ITHIN the simple Hückel molecular orbital (HMO) model, there are two energy-based stability criteria - the total $\pi$-electron energy and the energy difference between the highest occupied and lowest unoccupied molecular orbital, the so-called HOMO-LUMO gap. ${ }^{[1-3]}$ If the eigenvalues of the molecular graph are $\lambda_{1} \geq \lambda_{2} \geq \cdots \geq \lambda_{n}$,

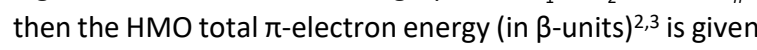
by

$$
E_{\pi}=2 \sum_{i=1}^{n / 2} \lambda_{i}
$$

whereas the eigenvalues $\lambda_{n / 2}$ and $\lambda_{n / 2+1}$ correspond, respectively, to the energies of HOMO and LUMO. Thus, the HOMO-LUMO gap is $\Delta_{H L}=\lambda_{n / 2}-\lambda_{n / 2+1}$, which for alternant hydrocarbons (including benzenoids), because of $\lambda_{n / 2+1}=-\lambda_{n / 2}$, is equal to

$$
\Delta_{H L}=2 \lambda_{n / 2} \text {. }
$$

In Eqs. (1) and (2), and later in the text, it is assumed that $n$, the number of $\pi$-electrons (and also the number of carbon atoms in the underlying conjugated molecule, and the number of vertices of the respective molecular graph) is even. If $n$ were odd, then the definition of the total $\pi$-electron energy would have to be modified, whereas the concepts of HOMO and LUMO would lose their meanings.

The total $\pi$-electron energy is related to the thermodynamic stability of the underlying conjugated compounds, for details see. ${ }^{[4,5]}$ The structure-dependency of $E_{\pi}$ is nowadays well understood, see $e^{[5-7]}$ and the references cited therein.

The HOMO-LUMO gap is considered as a measure of reactivity of the respective conjugated system. Although its structure-dependency has been much studied, ${ }^{[8-13]}$ George Hall's claim ${ }^{[14]}$ that "the variation in $\Delta_{H L}$ from molecule to molecule follows too complicated a pattern to be summarized in general rules" is still applicable.

Benzenoid hydrocarbons are the class of polycyclic conjugated molecules to which the predictions of the HMO model best agree with experimentally determined facts. The topological properties of this class of compounds have been studied in due detail. ${ }^{[5,15,16]}$ It is worth mentioning that Edward Kirby also did significant contributions to this field. ${ }^{[17-20]}$

It is reasonable to expect that $E_{\pi}$ and $\Delta_{H L}$ are somehow related. However, very little is known on such

(cc) BY This work is licensed under a Creative Commons Attribution 4.0 International License. 
hypothetical relation. In view of Hall's doubts, ${ }^{[14]}$ it may well be that its exact mathematical form will never be established.

In the paper ${ }^{[21]}$ the approximation

$$
E_{\pi} \approx A \sqrt{2 m n-x n\left(\frac{2 m}{n}-\frac{\Delta_{H L}}{2}\right)^{2}}+B
$$

was put forward, where $A, B$, and $x$ are fitting parameters. In the paper[22] another expression of this kind was considered:

$$
E_{\pi} \approx A\left(\frac{\Delta_{H L}}{2}+\sqrt{(n-1)\left(2 m-\frac{\Delta_{H L}^{2}}{4}\right)}\right)+B
$$

where $A$ and $B$ are fitting parameters (different from those in Eq. (3)). In formulas (3) and (4), and later in this text, $n$ denotes the number of vertices and $m$ the number of edges of the underlying molecular graph (i.e., the number of carbon atoms and carbon-carbon bonds of the respective conjugated molecule).

The approximations (3) and (4) are prohibitively complicated. The fact that their mathematical forms are completely different casts doubts on their reliability. We now offer a significantly simpler formula:

$$
E_{\pi} \approx\left(A_{0}+B_{0} \sqrt{\Delta_{H L}}\right) \sqrt{2 m n}
$$

which is based on the classical McClelland approximation $^{[23]}$ and a recently established result by Oboudi. ${ }^{[24]}$

\section{MCCLELLAND AND OBOUDI INEQUALITIES}

The upper bound (6) for total $\pi$-electron energy,

$$
E_{\pi}<\sqrt{2 m n}
$$

established by McClelland ${ }^{[23]}$ in 1971 , is one of the classical results of chemical graph theory.

Its practical value lies in the fact (also established by McClelland) ${ }^{[23]}$ that

$$
E_{\pi} \approx A \sqrt{2 m n}
$$

is a very accurate approximation, especially for benzenoids. Here, again, $A$ is a fitting parameter. Formula (7) was tested in due detail[5,25] and found to be better than any other $(n, m)$-type approximation.

For molecular graphs with even number of vertices,

$$
\left(\lambda_{1}-\lambda_{i}\right)\left(\lambda_{n / 2}-\lambda_{i}\right) \leq 0
$$

holds for all $i=1,2, \ldots, n / 2$, and the inequality is strict for at least some value of $i$. (In the case of benzene, $\left(\lambda_{1}-\lambda_{i}\right)\left(\lambda_{n / 2}-\lambda_{i}\right)=0$ holds for all $i=1,2, \ldots, n / 2$, but this is the only molecular graph with such an exceptional property.). Therefore, except for benzene,

$$
\sum_{i=1}^{n / 2}\left(\lambda_{1}-\lambda_{i}\right)\left(\lambda_{n / 2}-\lambda_{i}\right)<0
$$

i.e.,

$$
\frac{n}{2} \lambda_{1} \lambda_{n / 2}-\left(\lambda_{1}+\lambda_{n / 2}\right) \sum_{i=1}^{n / 2} \lambda_{i}+\sum_{i=1}^{n / 2}\left(\lambda_{i}\right)^{2}<0 .
$$

For alternant conjugated hydrocarbons (including benzenoids), $\sum_{i=1}^{n / 2}\left(\lambda_{i}\right)^{2}=m$. Then, in view of Eqs. (1) and (2),

$E_{\pi}>\frac{\lambda_{1} \lambda_{n / 2} n+2 m}{\lambda_{1}+\lambda_{n / 2}} \geq \frac{2 \sqrt{\lambda_{1} \lambda_{n / 2}}}{\lambda_{1}+\lambda_{n / 2}} \sqrt{2 m n}=\frac{2 \sqrt{2 \lambda_{1} \Delta_{H L}}}{2 \lambda_{1}+\Delta_{H L}} \sqrt{2 m n}$

and we arrive at Oboudi's lower bound. [24]

It is interesting to note that in Eq. (8), the McClelland term $\sqrt{2 m n}$ is multiplied by the ratio of the geometric and arithmetic means of $\lambda_{1}$ and $\lambda_{n / 2}$.

\section{RELATING $E_{\pi}$ AND $\Delta_{H L}$ FOR BENZENOID MOLECULES}

In this section we are concerned with Kekulèan benzenoid hydrocarbons, that is benzenoid systems possessing Kekulè structures and therefore a non-zero HOMO-LUMO gap. We require that these be polycyclic, that is to possess at least two mutually condensed benzene rings. Consequently, the smallest benzenoid hydrocarbon is naphthalene $(n=10)$, whereas benzene $(n=6)$ is excluded from consideration. Recall that the same has been done in our earlier studies of $\pi$-electron properties of benzenoid hydrocarbons. ${ }^{[5,21,22,25]}$

Bearing in mind McClelland's upper bound (6) and Oboudi's lower bound (8), we see that the true value of total $\pi$-electron energy must lie between these two estimates, i.e., it should be

$$
E_{\pi}=\left(A+\frac{2 \sqrt{2 \lambda_{1} \Delta_{H L}}}{2 \lambda_{1}+\Delta_{H L}} B\right) \sqrt{2 m n}
$$

for some pertinently chosen values $A$ and $B$. Note that for $B=0$, formula (9) reduces to the original McClelland approximation (7)

In the case of benzenoid systems (as well as practically all molecular graphs), $\Delta_{H L}$ is at least one order of magnitude smaller than the spectral radius $\lambda_{1}$. Therefore, $2 \lambda_{1}+\Delta_{H L} \approx 2 \lambda_{1}$ and thus Eq. (9) is simplified as

$$
E_{\pi}=\left(A+\sqrt{\frac{2 \Delta_{H L}}{\lambda_{1}}} B\right) \sqrt{2 m n} .
$$

The spectral radius of benzenoid systems varies in a 
very narrow range. Thus, for naphthalene $\lambda_{1}=2.3$ (minimum) and for ovalene $\lambda_{1}=2.7$ (close to maximum). Bearing this in mind, we can additionally simplify formula (10), arriving to Eq. (5).

\section{TESTING FORMULA (5)}

Same is in our earlier works, $[5,21,22,25]$ formula (5) was tested on the standard sample of 105 Kekulèan benzenoid hydrocarbons from the book of Zahradnik and Pancir. ${ }^{[26]}$ By least-squares fitting we found $A_{0}=0.889 \pm 0.001$ and $B_{0}=0.025 \pm 0.005$, with correlation coefficient $R=$ 0.99994 , average relative error $0.177 \%$, and maximal relative error $1.01 \%$. Thus, formula (5) has nearly the same precision as the earlier proposed approximations (3) and (4), but its advantage over (3) and (4) is in its simplicity. In contrast to (3) and (4), formula (7) reveals a straightforward connection between the two HMO stability criteria and its simple algebraic form will be easily acceptable by the chemistry community, especially by chemistry students.

\section{REFERENCES}

[1] A. Streitwieser, Molecular Orbital Theory for Organic Chemists, Wiley, New York, 1961. https://doi.org/10.1149/1.2425396

[2] A. Graovac, I. Gutman, N. Trinajstić, Topological Approach to the Chemistry of Conjugated Molecules, Springer, Berlin, 1977. https://doi.org/10.1007/978-3-642-93069-0

[3] C. A. Coulson, B. O'Leary, R. B. Mallion, Hückel Theory for Organic Chemists, Academic Press, London, 1978.

[4] I. Gutman, O. E. Polansky, Mathematical Concepts in Organic Chemistry, Springer, Berlin, 1986. https://doi.org/10.1007/978-3-642-70982-1

[5] I. Gutman,Topics Curr. Chem. 1992, 162, $29-63$. https://doi.org/10.14452/MR-043-10-1992-03_3

[6] I. Gutman, B. Furtula, Croat. Chem. Acta 2017, 90, 359-360. https://doi.org/10.5562/cca3189

[7] I. Redžepović, S. Marković, B. Furtula, MATCH Commun. Math. Comput. Chem. 2019, 82, 663-678.

[8] J. Aihara, J. Phys. Chem. A 1999, 103, 7487-7495. https://doi.org/10.1021/jp990092i
[9] Y. Ruiz-Morales, J. Phys. Chem. A 2002, 106, 1128311308. https://doi.org/10.1021/jp021152e

[10] P. W. Fowler, T. Pisanski, MATCH Commun. Math. Comput. Chem. 2010, 64, 373-390.

[11] D. J. Klein, Y. Yang, D. Ye, Proc. R. Soc. (London) A 2015, 471, 20150183.

https://doi.org/10.1098/rspa.2015.0183

[12] H. Chen, F. Zhang, MATCH Commun. Math. Comput. Chem. 2018, 80, 407-414.

[13] X. Li, Y. Weng, MATCH Commun. Math. Comput. Chem. 2019, 82, 459-468.

[14] G. G. Hall, Mol. Phys. 1977, 33, 551-557. https://doi.org/10.1080/00268977700100471

[15] I. Gutman, S. J. Cyvin, Introduction to the Theory of Benzenoid Hydrocarbons, Springer, Berlin, 1989. https://doi.org/10.1007/978-3-642-87143-6

[16] I. Gutman, Topics Curr. Chem. 1992, 162, 1-28. https://doi.org/10.1007/BFb0018561

[17] E. C. Kirby, J. Math. Chem. 1990, 4, 31-46. https://doi.org/10.1007/BF01170002

[18] E. C. Kirby, J. Chem. Soc. Faraday Trans. 1990, 86, 447-452. https://doi.org/10.1039/FT9908600447

[19] Y. Jiang, W. Yu, E. C. Kirby, J. Chem. Soc. Faraday Trans. 1991, 87, 3631-3640. https://doi.org/10.1039/FT9918703631

[20] E. C. Kirby, J. Math. Chem. 1991, 8, 77-87. https://doi.org/10.1007/BF01166925

[21] I. Gutman, S. Radenković, S. Đorđević, I. Ž. Milovanović, E. I. Milovanović, Chem. Phys. Lett. 2016, 649, 148-150. https://doi.org/10.1016/j.cplett.2016.02.051

[22] I. Gutman, S. Radenković, S. Đorđević, I. Ž. Milovanović, E. I. Milovanović, J. Math. Chem. 2017, 55, 1934-1940. https://doi.org/10.1007/s10910-017-0772-6

[23] B. J. McClelland, J. Chem. Phys. 1971, 54, 640-643. https://doi.org/10.1063/1.1674889

[24] M. R. Oboudi, Linear Algebra Appl. 2019, 580, 384395. https://doi.org/10.1016/j.laa.2019.06.026

[25] I. Gutman, T. Soldatović, MATCH Commun. Math. Comput. Chem. 2001, 44, 169-182.

[26] R. Zahradnik, J. Pancir, HMO Energy Characteristics, Plenum Press, New York, 1970. https://doi.org/10.1007/978-1-4684-6078-0 